\title{
SOLVENT SENSOR ARRAYS FOR BINARY MIXTURES USING POLYMER-BASED CHEMIRESISTORS
}

\author{
S. V. Patel, M. W. Jenkins, R. C. Hughes, W. G. Yelton, A. J. Ricco, and S. A. Casalnuovo* \\ Microsensor R \& D Department and *Advanced Semiconductor Technology Department \\ Sandia National Laboratories, Albuquerque, NM 87185
}

\begin{abstract}
Low power, carbon-loaded polymer chemiresistors have been used to sense solvents over a wide range of solubility parameters. Polyethylene-vinylacetate (PEVA) and polyvinylalcohol (PVA) are two such polymers that have been used to sense binary mixtures of diisopropylmethylphosphonate (DIMP) and other solvents with water vapor at various temperatures. The VERI (Visual Empirical Region of Influence) technique is used as a method of pattern recognition to classify the solvents and distinguish them from water vapor. A method for the determination of solvent concentrations in a binary mixture of the solvent and relative humidity is presented.
\end{abstract}

\section{INTRODUCTION}

The development of a low-cost, low-power, "universal" solvent vapor sensing array requires several sensing elements that can sense a wide range of solvents. Analysis of the component elements is important to determine the best possible sensor or sensors for the classification and quantification of solvent vapor mixtures. Polymer-based chemiresistors have been used recently in the development of solvent sensor arrays.[1,2] Such an array would contain a minimum number of sensors capable of responding to the largest number of analytes possible. Therefore the array must contain chemically different sensor elements capable of responding to different analytes.

Such arrays have been used with surface acoustic wave (SAW) sensors[3], where the solubility parameter $(\delta)$ is used to identify polymers best suited for sensing the solvents of interest. The polymer and solvent combination with similar solubility parameters result in the strongest interaction and, in turn, the largest response. Unfortunately, SAW devices require expensive and high frequency AC modulated electronics for operation. Arrays of this type also require a method of pattern recognition to analyze the responses of several devices to various solvents.

The VERI (Visual Empirical Region of Influence) pattern recognition method for solvent classification has been used to determine the best sensors for quantification of solvent mixture composition. [4] Once the minimum number of required devices are determined, the training set data are analyzed to determine the concentrations of solvent and relative humidity.

\section{EXPERIMENTAL DETAILS}

The polymer films used as chemiresistors were deposited on several different platforms. Detailed descriptions of the substrates are presented in reference [1]. The first type was the interdigitated electrodes on quartz substrates. These were fabricated at Sandia National Labs using photolithography to pattern gold electrodes with a chrome adhesion layer. The electrode spacing was 8 microns and the resistance was measured in a two-point probe configuration. On the same device a set of widely spaced electrodes was deposited to measure resistance of films that might have an uncharacteristically low resistance across the 8 micron spacing. A second type of platform consisted of platinum electrodes with a titanium adhesion layer, on an oxidized silicon wafer. The platinum electrodes were arranged in a four-point probe configuration, with two inner and two outer electrodes. The inner electrodes had either a 100 or 50 micron spacing. All the electrodes made contact to large pads for external electrical contact. These data are the first reported" four-terminal measurements on this type of chemiresistor in which the bulk resistance can be separated from the contact resistance.

The carbon-loaded polymers were made by dissolution of both the polymer and a particular weight percent (e.g., $40 \mathrm{wt}$. \% is designated by " $-40-C$ ") of carbon particles (20-30 nm diameter) in a solvent such as water or chlorobenzene. The polymers used were polyisobutylene (PIB), polydiphenoxyphosphazine (PDPP), polyethylene-vinylacetate (PEVA), polyvinylalcohol in two forms, $88 \%$ (PVA) and 75\% (PVA75) hydroxylated. The carbon loading typically ranged from 25 to $40 \mathrm{wt}$. \%.

Films were deposited on substrates by either spin casting or pipetting the dissolved material directly onto the substrate. The pipette method usually led to films that were thicker than the spin cast films. In some cases a 5 micron filter was used to enhance the dispersion of the carbon particles, which tended agglomerate and form shorts between the narrowly spaced electrodes.

The devices were placed in a specially designed test fixture, which had spring-loaded Pogo ${ }^{\circledR}$ pins to contact the pads on the substrates. The fixture was attached to a gas manifold that had the capability of mixing several gases as well as two solvent vapor streams using nitrogen as the carrier gas through bubblers. Eleven solvent vapors were tested with varying solubility parameters. A constant total flow rate of $1000 \mathrm{sccm}$ was used for all experiments. The test fixture was placed in a constant temperature chamber which could be controlled to $\pm 0.1^{\circ} \mathrm{C}$ and heated to $60^{\circ} \mathrm{C}$. For the carbon-loaded polymers, a digital multimeter ( 7.5 digit resolution) was used to measure the film resistance.

\section{RESULTS AND DISCUSSION}

The sensor films were exposed several times to solvent vapors individually as well as simultaneously with water vapor to challenge the array for the most common binary mixture encountered in the field. Fig. 1 shows the percent resistance change response of five polymer composites normalized to the largest response for each device. At least one of the five chosen films responds to each analyte over the entire range of solvents from isooctane $(\delta=15.6)$ to water $(\delta=48)$. Using the VERI method of pattern recognition, the responses are plotted on the surface of a unit sphere. Fig. 2 contains the normalized and equalized [4] responses of three films to several pure solvents and binary mixtures of these solvents and water vapor. Each point is a different set of concentrations (water, solvent vapor). Using just three polymer composite films, several different solvent vapors and binary mixtures with water vapor can be distinguished visually. The sensor responses to an unknown vapor are compared 


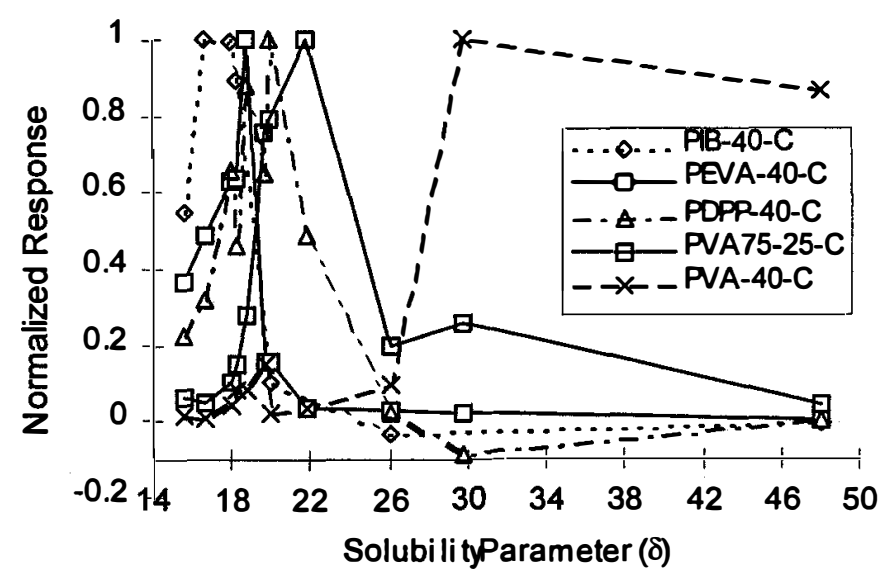

Fig. 1 Normalized response of carbon-loaded polymer films to 11 solvents spanning the range of solubility parameters from isooctane to water. The solvent vapor for each exposure was at $23^{\circ} \mathrm{C}$ and $P / P_{\text {sat }}=0.10$.

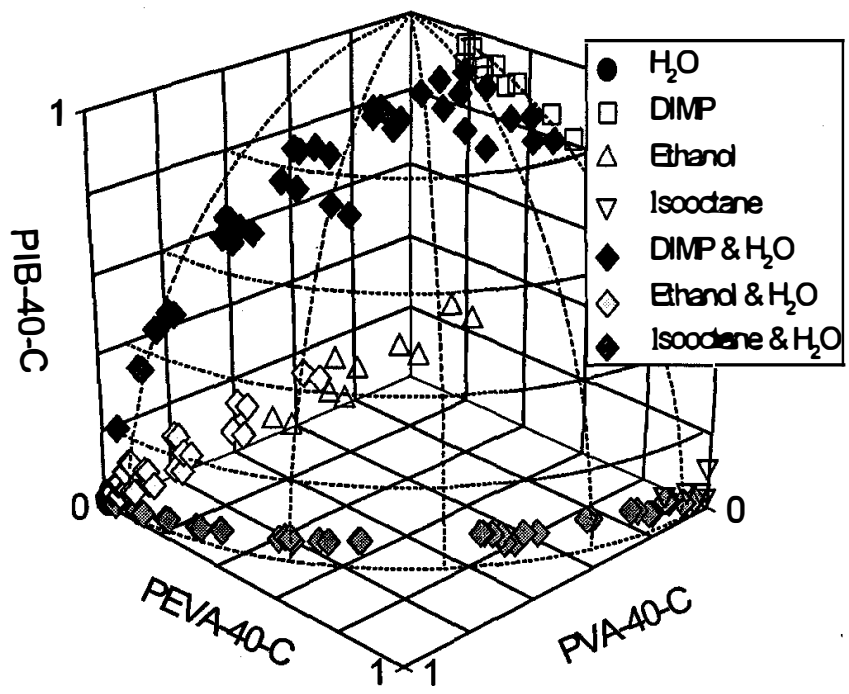

Fig. 2 Pattern recognition for distinguishing classes of solvents. Using three polymer composite films, several different solvent vapors can be distinguished visually. The sensors and vapors were at $23^{\circ} \mathrm{C}$.

with patterns, such as in Fig. 2, and once classification is achieved the concentrations of the water vapor and solvent can be determined from the training set data.

An example of such a binary training set is shown in Fig. 3 where the PVA-40-C and PEVA-40-C sensor data for a DIMP (diisopropylmethylphosphonate)/water vapor binary mixture are plotted. In this case, the DIMP concentration was varied with constant relative humidity in the background gas stream. Any new data point, representing an unknown mixture, will be surrounded by four points in the training set and the concentrations of both species can be estimated by interpolation. For a real sensor array, more data could be collected to fill in the gaps in the data set, and to determine the error or confidence level in each value.

The curvature of the isochemical lines in Fig. 3 shows that the responses of binaries are not simply superpositions of the response of the individual concentrations. The curvature means that the presence of one analyte can have a measurable effect on the sensor's response to the other analyte.

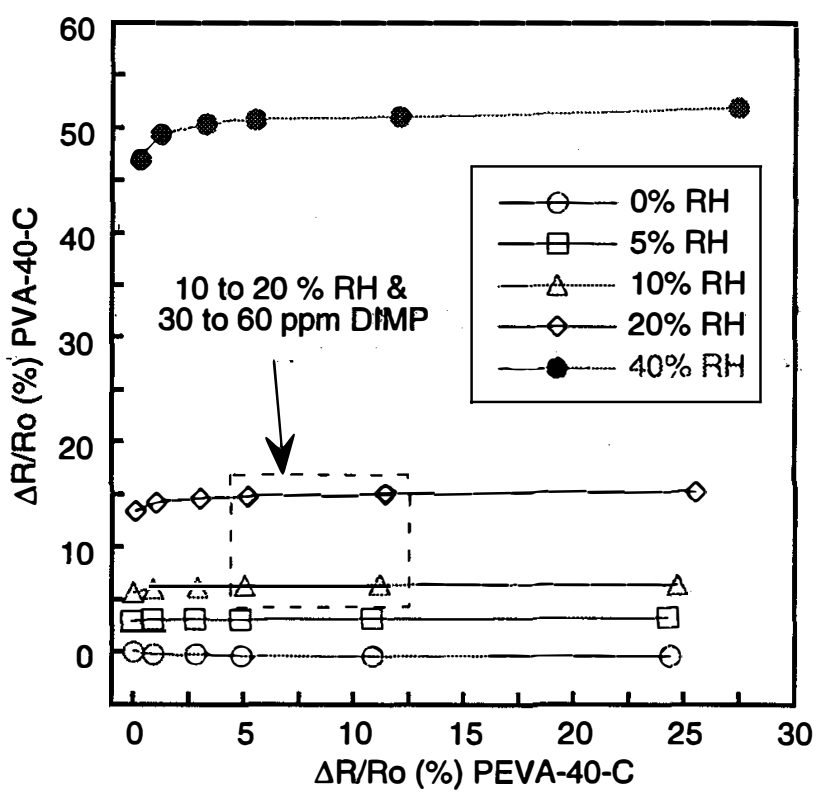

Fig. 3 The relative response of two sensors to binary mixtures of water and DIMP vapors. Water concentration is given as \% relative humidity $(R H)$. The DIMP concentration $\left(P / P_{\text {sat }}=0,1\right.$, $3,5,10,20 \%)$ was varied with constant relative humidity in the background gas stream. P/P sat for $100 \%$ DIMP at $23^{\circ} \mathrm{C}$, the temperature of the bubblers and sensors, is equal to 600 ppm.

\section{CONCLUSIONS}

Several carbon-loaded polymer films have been used as solvent sensors in an array. The array is capable of responding to a wide range of solvent vapors with widely varying solubility parameters. A method for the classification and quantification of binary mixtures of solvents is presented. This method relies on training data sets, which map out the regions of interest for watersolvent vapor pairs. This simplified version of the VERI method can be applied to any system of sensors, as well as for more than three sensor responses.

\section{REFERENCES}

1. R. C. Hughes, M.'P. Eastman, W. G. Yelton, A. J. Ricco, S. V. Patel, and M. W. Jenkins, "Application of the Solubility Parameter Concept to the Design of Chemiresistor Arrays", Technical Digest of the 1998 Solid-State Sensor and Actuator Workshop, Hilton Head Isl., SC, 6/7-11/98.

2. M. C. Lonergan, E. J. Severin, B. J. Doleman, S. A. Beaber, R. H. Grubbs, and N. S. Lewis, "Array-Based Vapor Sensing Using Chemically Sensitive, Carbon Black-Polymer Resistors", Chem. Mater., 8, 2298 (1996).

3. R. A. McGill, M. H. Abraham, and J. W. Grate, "Choosing Polymer Coatings for Chemical Sensors", Chemtech, 24, 27 (1994).

4. G. C. Osbourn, J. W. Bartholemew, A. J. Ricco and G. C. Frye, "VERI Pattern Recognition Applied to Chemical Microsensor Array Selection and Chemical Analysis", Accts. Chem. Res., 31, xxxx (1998). 